\title{
27 Schuld in der Notfallmedizin und in der Notfallseelsorge
}

\author{
Kerstin Lammer
}

\begin{abstract}
An einer Unfallstelle mit mehreren Verletzten hat sich die Notarzt entschieden: Welcher Patient wird zuerst versorgt? Während er diesen Patienten behandelt, stirbt ein anderer, unbehandelter. Der Arzt hat entscheiden müssen. Kollegen bestätigen, dass seine Entscheidung richtig war. Aber nun hat er Schuldgefühle - zu Recht? (Bsp. 1)

Ein Amok-Läufer hat aus Hass und Rache auf acht Menschen geschossen. Die Notfallseelsorgerin weiß zwar: Auch der Täter hat Anspruch auf Seelsorge; er hat sie womöglich besonders nötig. Aber wie kann sie mit ihren inneren Vorbehalten umgehen? Kann sie dem Mann helfen? (Bsp. 2)

Eine Frau wurde nachts auf dem Weg durch einen Park vergewaltigt und durch Schläge, Tritte und Würgen schwer verletzt. Sie sagt: „Ich bin selbst schuld. Warum musste ich auch im Dunkeln da entlang gehen?“. Das Notfallteam möchte ihr ihre nicht realitätsgerechten Schuldgefühle ausreden, aber sie hält hartnäckig daran fest - wozu? (Bsp. 3)
\end{abstract}

Notfallmedizin und Notfallseelsorge arbeiten mit Menschen in existentiellen Grenzsituationen, die für Betroffene und Helfende oft emotional überfordernd sind. Psychoedukation gehört daher zu Recht längst zum professionellen Standard. Ob Trauma, Trauer, Suizidalität etc. - professionell Helfende kennen psychische Prozesse und Bewältigungshilfen in Krisenfällen; sie lernen, sich selbst damit auseinanderzusetzen und Patient/innen und deren Zugehörige darüber aufzuklären.

Ethische Edukation dagegen wird Helfenden wie Betroffenen selten angeboten. Doch Schuld und Schuldgefühle spielen in vielen Notfällen explizit oder implizit eine wichtige Rolle. Für solche Fälle brauchen professionell Helfende zuallererst selbst eine 
ethisch-sittliche Orientierung. Denn auch, wenn es nicht ihr Anliegen ist, zu bewerten - sie tun es unwillkürlich. Agieren mit unbewussten Halburteilen und diffusunsicheren moralischen Gefühlen (oder deren Abwehr) schafft aber Unsicherheiten. Wer das Geschehene bewusst einordnet und bewertet, kann seinen Patient/innen ein ruhigeres und klareres Gegenüber sein. Notfallteams sollten deshalb selbst eine ethische Edukation haben. Darüber hinaus haben innerhalb der Notfallteams die Seelsorgenden die fachliche Expertise, anderen Beteiligten eine ethische und eine (theologisch-)anthropologische Edukation anzubieten.

Dieser Artikel will Folgendes leisten:

1. Ethische Orientierung: Was ist Schuld und wie ist Schuld zu bewerten? (Sechs Kategorien von Schulderleben mit und ohne Verschulden);

2. Theologisch-konfessionelle Orientierung: Modelle von Schuldentlastung und Schuldbewältigung;

3. Psychologische Orientierung: zum positiven Sinn „falscher“ oder „neurotischer" Schuldgefühle bei Menschen, die keine wirkliche Schuld haben;

4. Theologisch-existentiale Orientierung: das Maß des Menschseins - heilsamer Umgang mit Schulderleben;

5. Interprofessionelle Orientierung: zu Rolle und Funktion der Seelsorge.

\subsection{Ethische Orientierung: Was ist Schuld, und wie ist Schuld zu bewerten? Sechs Kategorien von Schulderleben mit und ohne Verschulden}

Seit dem 19. Jahrhundert hat „Schuld“ in Theologie und Gesellschaft keine gute Presse. Zu Recht hat die Religionskritik angeprangert, dass die Kirchen jahrhundertelang Schindluder mit der Schuldangst der Menschen getrieben hatten. Die Predigt von Sünde und Schuld beschrieb Friedrich Nietzsche als Erfindung der Priester zur Herrschaft über die Menschen. Die neuere Theologie, allzumal die evangelische, hat von Nietzsche ${ }^{16}$ gelernt, dass Christen erlöster aussehen müssten, und von Paul Tillich ${ }^{17}$, dass das Zeitalter des gequälten, erschrockenen Cewissens lange vorbei sei, abgelöst vom Zeitalter des gekränkten Gewissens. Nicht mehr die Angst vor Verdammung, sondern statt dessen die Angst vor Leere und Sinnlosigkeit bilde die Erlebnisstruktur des modernen Menschen; nicht mehr die Frage nach Schuld und Vergebung, sondern statt dessen die Fragen nach Lebensfülle, Selbstverwirklichung, Zugehörigkeit, Beheimatung und Sinn - psychologisch gesehen also nicht mehr der Konflikt mit dem Über-Ich, sondern eher narzisstische Thematiken wie der Konflikt mit dem eigenen Größen-Selbst.

Als neue Form der christlichen Rechtfertigungsbotschaft wurde „nicht [mehr] die Vergebung von Sünde und Schuld als vielmehr die Befreiung von falschen Schuldgefühlen“ verstanden. ${ }^{18}$ Und so haben wir uns angewöhnt, zwischen objektiver „Schuld“ und subjektivem „Schuldgefühl“ zu unterscheiden, und, wenn uns Schuldgefühle be-

16 Nietzsche, Friedrich, Also sprach Zarathustra. Ein Buch für Alle und Keinen (1883-1885), Nachdruck

17 Tillich, Paul, Mut zum Sein, Stuttgart, 3. Aufl. 1953, S. 33ff. (am 1952).

18 Körtner, U., Sündenvergebung und Schuldübernahme in der Seelsorge, in: WzM 58, 2006, 259-269, hier 261, Einfügung und Hervorhb. durch die Autorin. 
gegnen, zuerst danach zu fragen, ob dieses Gefühl überhaupt „angemessen“ sei, d.h.: ob denn dahinter wirklich ein echtes schuldhaftes Verhalten stehe, oder ob das subjektive Schuldgefühl nicht unrealistisch, ja neurotisch und der betroffene Mensch möglichst davon abzubringen sei. ${ }^{19}$

Mit derTendenz, Schulderleben als etwas Uneigentliches einzuordnen und wegberaten zu wollen, sind Helfende jedoch mitunter zu schnell bei der Hand.

Was, wenn wirkliche Schuld vorliegt? Können wir das selber aushalten? Und haben wir etwas anzubieten, das den Betroffenen hilft, ihre Schuld auszuhalten? So, dass die Betroffenen kein reines, aber ein getröstetes Gewissen bekommen?

Und was, wenn subjektive Schuldgefühle zwar aus ethisch-moralischer Sicht unsinnig sind, weil objektiv kein schuldhaftes Fehlverhalten vorliegt, sie aber eine andere positive Funktion haben, weil ihnen andere relevante Realitäten zugrundeliegen (psychischen Realitäten, Beziehungsrealitäten, spirituelle Realitäten)? Können „falsche" Schuldgefühle sinnvoll sein?

Womöglich nehmen wir unseren Klient/innen und Patient/innen etwas Wesentliches, wenn wir ihnen ihr Schulderleben nehmen wollen. Stattdessen plädieren wir hier für einen weder dramatisierenden noch marginalisierenden, sondern akzeptierenden Umgang mit Schulderleben.

\section{Was ist Schuld?}

Die christliche Dogmatik verhandelt die Schuldthematik gemeinhin unter dem Begriff der „Sünde“. Sünde und Schuld werden dabei oft synonym gebraucht. Oder ihr Gebrauch wird durch den Beziehungspartner bzw. Adressaten unterschieden: Sündig ist man gegenüber Gott, schuldig ist man gegenüber dem Mitmenschen.

Schuld wird traditionell (seit der Satisfaktionslehre des Anselm von Canterbury im 11. Jh.) in Tatschuld und Existentialschuld unterteilt. Die Tatschuld (lat. culpa) bezeichnet das Verschuldete. Durch Normübertretung, durch Tun des Falschen oder Bösen hat man moralische Schuld auf sich geladen und hat Anlass, mit schlechtem Gewissen zu bekennen: „mea culpa, mea culpa, mea maxima culpa (meine Schuld, meine Schuld, meine große Schuld)“. Dagegen bezeichnet die Existentialschuld (lat. debitum) das Geschuldete, eine Schuld nicht durch die Tat, sondern durch das Sein: Man bleibt dem Mitmenschen, sich selbst und Gott etwas schuldig, weil man nicht ist, was man sein könnte oder sollte, weil man an die Grenzen seiner Möglichkeiten stößt, weil man handeln muss und Handeln immer unerwünschte Folgen haben kann, weil oft „Leben nur auf Kosten anderen Lebens möglich“20 ist. Die Unentrinnbarkeit dieser Seinsschuld wird in der christlichen Tradition mit dem Begriff „Erbsünde“ angezeigt. Dabei ist der springende Punkt: Diese Art von Schuld ist unvermeidlich mit dem

19 Erst in jüngerer Zeit erfuhr die Marginalisierung von Schuld und Schuldgefühlen eine Gegenbewegung. Dier katholische Kirche prangert den Wertewandel in der Gesellschaft an: Was früher als Todsünde galt, werde im Zeitalter des Hädonismus als Tugend betrachtet: Aus Völlerei sei Gaumenfreude, aus Unkeuschheit Sex und Sinnlichkeit, aus Habgier Luxus und aus Hoffart Selbstsicherheit geworden; ein „,heimlicher Unschuldswahn“ breite sich aus, „, mit dem wir Schuld und Versagen, wenn überhaupt, immer nur bei ,den anderen' suchen ..." (Synode der Bistümer 1976, zit. nach Sievenich, M., Schuld und Vergebung. Grundthema und Anfrage, in: WzM 57, 2005, 298-308, hier 304). Nachdem der Psychoanalytiker Horst Eberhard Richter die „Krankheit der Unverantwortlichkeit“ in der Gesellschaft diagnostiziert hatte, forderte sein Kollege Thomas Auchter eine Rückkehr „von der Unschuld zur Verantwortung" (vgl. Richter HE, Wer nicht leiden will, muss hassen, Hamburg 1993, S. 121; Auchter T, Von der Unschuld zur Verantwortung, in: 1996, S. 41-138)

20 Klessmann, M., Pastoralpsychologie. Ein Lehrbuch. Neukirchen-Vluyn 2004, 598. 
Menschsein an sich gegeben - nicht erst, wenn und weil wir böse wären, sondern schon, weil wir begrenzt sind. Damit vertreten wir hier einen postkonventionellen, transmoralischen Begriff von Schuld und Sünde. ${ }^{21}$

Die traditionelle Unterscheidung zwischen Tatschuld und Seinsschuld möchten wir weiter ausdifferenzieren, indem wir nach eigenem Dafürhalten sechs Fallgruppen von Schulderleben unterscheiden, die uns in Notfallmedizin und Notfallseelsorge begegnen. ${ }^{22}$

\section{Drei Fallgruppen von Schulderleben ohne Verschulden}

Schulderleben ohne tätiges Verschulden lässt sich in drei Fallgruppen kategorisieren:

1. Schulderleben als psychische Verschiebung unerträglichen anderen Erlebens („lieber schuldig als ...")

Häufig wird Schulderleben entwickelt, um ein anderes, noch schwerer auszuhaltendes Erleben zu überdecken; es handelt sich dabei um eine Form von Verschiebung.

Dieser Mechanismus liegt beim eingangs genannten Fallbeispiel der Frau vor, die sich selbst die Schuld für die gewalttätigen Übergriffe auf sie gibt. (Bsp. 3).

Weitere Beispiele sind:

Die Ehefrau hat im Tag und Nacht am Sterbebett ihres Mannes gewacht; morgens geht sie sich kurz frischmachen, und ausgerechnet in diesen einzigen wenigen Momenten ihrer Abwesenheit stirbt ihr Mann. Sie fühlt sich schuldig an seinem einsamen Tod. (Bsp. 4)

Ein Mädchen wurde jahrelang von seinem Vater missbraucht. „Der Papa hat mich so lieb“, sagt sie, „aber ich bin eben eine Schlampe.“

Lieber interpretieren sich die betroffenen Menschen als schuldig als als ohnmächtig, ausgeliefert oder ungeliebt. Das Schulderleben dient der Abwehr anderer, für die Betroffenen unerträglicher Gefühle.

21 Was bedeutet „ein postkonventioneller, transmoralischer Sündenbegriff“? Für theologisch Interessierte sei hierzu ein kleiner dogmatischer Exkurs erlaubt: Mit Wilfried Joest meine ich, dass eine konventionelle Sühnopfer-Theologie überholt ist. Zugespitzt möchte ich formulieren: Paulus hat mit seinem viel zititerten Satz „Der Tod ist der Sünde Sold“ (Röm 5,12) unrecht. Dass der Tod die Strafe oder der Preis für die Sünde des Menschen sei, ist exegetisch und dogmatisch falsch. Es ist exegetisch falsch, denn laut Schöpfungsbericht müssen die Menschen nicht sterben, weil sie sündig sind, sondern weil sie geschöpflich, also endlich und sterblich sind: „Von Erde bist du genommen, zu Erde wirst du wieder werden“ (Gen 3,19). Und es ist dogmatisch falsch, denn der springende Punkt des Versöhnungs- und Rechtfertigungswerks Jesu Christi ist - mindestens nach evangelischer Überzeugung gerade der, dass uns die Sünde nicht mehr zugerechnet wird, und dass wir dafür nicht mehr bestraft werden. Der Tod folgt also nicht aus der Sünde, sondern die Sünde folgt aus dem Tod. M.a.W.: Weil wir endlich, und das heißt begrenzt sind, können wir nicht anders, als sündig zu sein und schuldig zu werden, gewollt oder ungewollt. Aus unseren Begrenzungen und Fehlbarkeiten, aus unseren Schwächen, aus dem Konflikthaften und Unheilen in uns kommen wir nicht heraus, weil wir begrenzte Menschen sind (und nicht Gott). Deshalb müssen wir leiden, sterben und uns schuldig machen. Diesen Tatsachen gilt es bewusst ins Auge zu sehen. Wir können daraus nur von jenseits unserer selbst her erlöst werden. In diesem Sinne vertrete ich hier eine theologische Anthropologie der Begrenztheit.

22 In vielen dieser Fälle spielen auch andere Themen als Schuld eine große Rolle, Trauer z.B. oder Traumatisierung. Diese Themen klammere ich hier aus; ich habe sie anderenorts behandelt: vgl. Lammer, Kerstin: Trauer verstehen. Formen-Erklärungen-Hilfen, Heidelberg (Springer Medizin), 4. Aufl. 2013. Dies.: Den Tod begreifen. Neue Wege in der Trauerbegleitung, Neukirchen-Vluyn (Neukirchener), 6. Aufl. 2013. Und zum Thema Seelsorge mit Traumatisierten: Lammer, Kerstin, Kalter Schweiß auf dem Rücken. Seelsorge nach belastenden Ereignissen, in: dies., Beratung mit religiöser Kompetenz. Beiträge zu pastoralpsychologischer Seelsorge und Supervision, Neukirchen Vluyn (Neukirchener) 2012, 94-106. 
27.1 Ethische Orientierung: Was ist Schuld, und wie ist Schuld zu bewerten? Sechs Kategorien von Schulderleben mit und ohne Verschulden

2. Schuld als tragische Verstrickung (ohne eigenes Zutun) oder

3. Schuld als Überlebens-,,Schuld“:

Ein suizidaler Mann springt vor den ICE aufs Gleis, der Lokführer kann den Zug nicht mehr rechtzeitig zum Stehen bringen und überfährt den Mann. Ihn quälen Schuldgefühle. (Bsp. 5)

Ein Lehrer und eine Lehrerin machen mit ihrer Klasse eine Radtour, sie fährt vor den Kindern, er hinter ihnen. Ein betrunkener Autofahrer kommt entgegen, fährt die vorne fahrende Lehrerin an; sie stirbt. Der Lehrer fühlt sich schuldig, weil die Kollegin zu Tode kam, und nicht er. (Bsp. 6)

Beide Männer können nichts dafür, was geschehen ist, sie haben kein Verschulden. Und dennoch ist es schmerzvoll und beängstigend und schwer zu ertragen,

- dass ein anderer stirbt, weil ich gerade da bin, wo ich bin;

- dass ein anderer stirbt, während ich gerade da bin und nichts dagegen tun kann;

- dass es reiner Zufall ist, dass der Andere stirbt und ich lebe („Überlebensschuld“).

Das Schuldgefühl zeigt das Belastende solcher Erfahrungen an und die Beteiligung: Ich bin äußerlich irgendwie beteiligt am Schicksal des Anderen und ich spüre meine innere Beteiligung: Es ist mir nicht egal, ich habe Ehrfurcht vor seinem Leben und Tod.

In allen drei bis hierher skizzierten Fallgruppen haben die Klienten keinerlei Verfehlungen begangen, sie haben sich im moralisch-ethischen Sinne nicht schuldig gemacht. Dieses ethische Urteil ist klar, und es wird irgendwann für sie wichtig werden. Dennoch haben sie Anlass und Grund für ihr Schuldgefühl: Es macht zunächst Sinn - nicht ethisch, aber psychologisch oder spirituell oder beides (s.u.). Darauf ist seelsorglich einzugehen. Es zu übergehen, wäre ein Kunstfehler.

\section{Drei Fallgruppen tatsächlicher Schuld}

In den nächsten drei Fallgruppen liegt eine Tatschuld im eigentlichen Sinne des Verschuldens vor.

4. Schuld als Verantwortung für Folgen menschlichen Versagens: (falsches Tun ohne falsches Wollen)

Ein LKW-Fahrer hat an einer roten Ampel nicht gebremst und ist in eine Fußgängergruppe gerast, die die grüne Fußgängerampel überquerte. Es gibt Tote und Verletzte. „Ich habe gesehen, dass die Ampel rot war", sagt er, „ich weiß auch nicht, warum ich nicht gebremst habe." (Bsp. 7)

Im o.a. Fall war der betroffene Mann vielleicht übermüdet, verliebt, traurig, abgelenkt, hat an irgendetwas Anderes gedacht und deshalb nicht reagiert - alles normale, menschliche Dinge, die jedem von uns täglich passieren können und passieren. Aber nicht bei jedem von uns haben sie so schlimme Folgen. Der Mann ist nicht böse, aber er ist schuldig am Leiden und Sterben der Opfer seiner Unaufmerksamkeit. Versagen ist menschlich, aber er trägt die Verantwortung für die Folgen seines Versagens. Die Schwere dieser Last gilt es zu würdigen, nicht zu ermäßigen. 
5. Schuld als Verantwortung für unvermeidbare Folgen ethischer Entscheidungen (richtiges Tun mit falschen Folgen)

Für diese Fallgruppe wurde eingangs das Beispiel des Notarztes genannt, der sich entscheiden musste, in welcher Reihenfolge er schwer verletzte Menschen behandelt (Bsp. 1). Er hat nichts falsch gemacht, aber seine Entscheidung für die Behandlung des ersten und gegen die des zweiten Patienten hat möglicherweise zum Tod des zweiten geführt. Fälle wie dieser zeigen das unvermeidliche Dilemma: Wir können nicht unschuldig bleiben, wenn wir verantwortlich leben wollen. Wenn wir nicht handlungsunfähig werden wollen, müssen wir zu Entscheidungen mit möglicherweise unerwünschten negativen Folgen und zur Übernahme der Schuld an diesen Folgen bereit sein. Wer Verantwortung übernimmt, kann nicht heilig oder unschuldig bleiben; er muss mit schlechten Folgen seines Handelns leben und dafür einstehen. Die ethische Anforderung (man könnte auch sagen: die moralische Pflicht) ist hier also: nicht unschuldig bleiben wollen, sondern: bereit sein zur Übernahme von Verantwortung und Schuld. Es ist unverantwortlich, das zu vermeiden. Das hat Luther gemeint, als er sagte: peccate fortiter (sündigt kräftig).

Wenn Schuld als Folge ethischer Entscheidungen auftritt, ist es seelsorglich geboten,

v das Aufgeben von Unschuldswünschen als verantwortliche Verzichtsleistung zu würdigen und

- die Übernahme von Schuld als sittlich gute Entscheidung zu würdigen.

6. Schuld als Verantwortung für die Folgen falschen/bösen Wollens oder Tuns:

Ein LKW-Fahrer hat sich fahrlässig nach Alkoholgenuss ans Steuer gesetzt, die Kontrolle über das Fahrzeug verloren und einen Unfall verursacht. Ein Mensch ist tödlich, einer sehr schwer, der LKW-Fahrer selbst leicht verletzt. (Bsp. 8)

Ein Amokläufer hat in einer Schule aus Hass und Rache wahllos auf 16 Menschen geschossen (Bsp. 9).

Ein Erpresser hat aus Habgier eine Geisel genommen; bei einem Schusswechsel mit der Polizei sind er selbst und ein Polizist verletzt worden. (Bsp. 10)

Diese letzte Fallgruppe kommt in Notfallmedizin und Notfallseelsorge zum Glück am seltensten vor. Mit diesen Fällen ist am schwierigsten umzugehen, weil sie uns mit der menschlichen Bosheit und mit der Frage der Annahme des Unannehmbaren, des Verwerflichen konfrontieren. Seelsorgende stoßen hier an die Grenzen ihrer Fähigkeit zur Empathie und zur mitgeschöpflichen Solidarität mit den Tätern, wenn sich Empathie und Solidarität mit den Opfern in den Vordergrund drängen. Das beratungsethische Gebot der Allparteilichkeit ist hier am schwersten durchzuhalten. Kognitiv ist ihnen klar: Sie sollen seelsorglich unterscheiden zwischen der Tat (die unannehmbar ist) und der Person, die sie begangen hat. Denn die Person ist mehr als nur Täter, er ist Mensch, und als solcher Teil der menschlichen Schicksals- und Heilsgemeinschaft. Ihm gilt die Zusage der Cnade und Annahme Gottes genauso wie dem Opfer und wie dem Seelsorger. Der Täter hat also gleiche Recht auf seelsorgliche Zuwendung wie alle anderen. Emotional können Seelsorgende dieses theologische Postulat aber nicht immer umsetzen; es ist insbesondere dann schwierig, wenn Täter keine Schuldeinsicht und keine Reue zu erkennen geben. Dann kann es passieren, dass man dem 
Täter gegenüber zugeben muss: Ich glaube, dass Cott Dich annimmt, aber ich kann es nicht. Man bleibt dem Täter dann schuldig, wozu man ihm als Seelsorger/in und als Christ/in verpflichtet ist. Diese Erkenntnis kann allerdings ein neuer Anfang sein, denn damit sitzen beide Beteiligten im selben Boot des Schuldig-Seins.

\subsection{Theologisch-konfessionelle Orientierung: Modelle von Schuldentlastung und Schuldbewältigung}

Zur Entlastung von Schuld kennen Bibel und kirchliche Tradition unterschiedliche Wege. Sie werden - zugegebenermaßen stark vereinfachend - in zwei Typen eingeteilt:

1. das Ausgleichs- oder Wiedergutmachungsmodell, eher katholisch rezipiert und ausgestaltet, und

2. das Solidaritätsmodell, eher evangelischer Theologie entsprechend.

zu 1.: Das Ausgleichs- oder Wiedergutmachungsmodell finden wir in der Bergpredigt Mt 6. 1-18; es wird in den drei Schritten Fasten - Beten - Almosengeben vollzogen. Verstehen wir dies eher existentiell als moralisch, hat es in etwa folgenden Sinn: Die Schuld bindet den Schuldigen in unguten Verstrickungen; seine Beziehungen zu Mitmenschen und zu Gott sind gestört. Das Fasten als Verzicht soll ihn von falschen Abhängigkeiten und von der eigenen Ungerechtigkeit frei machen. Und es soll ihn frei machen zu neuer Bezogenheit. Das Beten soll den Bezug zu Gott neu herstellen, das Almosengeben den Bezug zum Mitmenschen. Wem man sich vorher entzogen hat, dem wendet man sich jetzt wieder zu, wem man vorher durch seine Schuld etwas genommen hat, dem gibt man jetzt wieder etwas zurück. Man sucht die durch die Schuld entstandenen Ungleichgewichte exemplarisch wieder auszugleichen.

Einen ähnlichen Dreischritt kennt die Scholastik: Auf die contritio cordis, d.h. die Reue des Herzens, folgt die confessio oris, d.h. das mündliche Bekennen, und dann die satisfactio operi, also die Taten der Genugtuung - eine ausgleichende Wiedergutmachung durch gute Taten.

Viele Menschen mit Schuldgefühlen haben ein Bedürfnis nach einer solchen ausgleichenden Buße und möchten etwas Gutes tun, um wenigstens einen Teil ihrer Schuld abzutragen. Auch von Geschädigten und von der sozialen Gemeinschaft wird oft eine Art von Täter-Opfer-Ausgleich gefordert. Hilfreich sind am Ausgleichs- oder Wiedergutmachungsmodell vor allem zwei Aspekte:

- Die Schuld wird darin als Beziehungsgeschehen verstanden. Es wird deutlich, dass Beziehungsarbeit geleistet werden muss, um die Schräglage, die die Schuld in die Beziehung gebracht hat, wieder auszugleichen.

- Es wird deutlich, dass Vergebung nicht als ein billiges Geschäft zu verstehen ist, sondern als ein längerer Prozess in mehreren Schritten, die selten schmerzfrei verlaufen. ${ }^{23}$

Nachteilig an diesem Modell ist, dass es ein wesentliches Problem nicht löst, sondern u.U. noch steigert, wenn sich die Vergeblichkeit des aufgezeigten und beschrittenen

23 Auf beide Aspekte hat schon Michael Klessmann hingewiesen; vgl. in: ders., Seelsorge. Ein Lehrbuch, Neukirchen Vluyn 2008, 241. 
Weges zeigt: Ein Schuldausgleich ist oft auf einer Handlungsebene nicht mehr herzustellen. Die Erfahrung, dass das geschehene Übel nicht wieder gut zu machen ist (Luther spricht hier auch vom „Schmerz der Sünde“), ist ein existentielles bzw. spirituelles Problem, das nicht durch Aktivität, sondern durch relationale Rezeptivität zu lösen ist, d.h.: nicht auf dem Weg des Handelns, sondern nur auf dem Weg des Glaubens bzw. der Annahme der eigenen Systembedingungen als begrenzter, unvollkommener und vergebungsbedürftiger Mensch in einer unvollkommenen Welt.

zu 2.: Das von mir so genannte Solidaritätsmodell betont nicht einen kategorialen (und auszugleichenden) Unterschied zwischen Tätern und Opfern, sondern deren prinzipielle Gleichheit. Hier ist davon auszugehen, dass alle Menschen schuldig werden, weil sie gar nicht anders können (s.o.), und dass wir Menschen von daher einander die Vergebung schulden, derer wir alle selbst bedürfen. Hierbei handelt es sich m.E. um die notwendige zwischenmenschliche Konsequenz aus der christlichen Lehre von Sünde und Rechtfertigung: Wir identifizieren uns als selbst Schuldige mit anderen Schuldigen - und das ist befreiend. Wir sondern keine Sündenböcke mehr aus, denn wir sitzen alle im selben Boot. In der Gemeinschaft der Begrenzten und Unvollkommenen ist Schuld für alle unvermeidlich und alle brauchen Vergebung; Vergebung ist daher etwas, das man zu nehmen und zu geben hat (vgl. die Vaterunser-Bitte nach Mt 6,12 „... und vergib uns unsere Schuld, wie auch wir vergeben unseren Schuldigern“).

Seelsorglich wichtig ist daran der Aspekt der Verbundenheit, auf den unten noch zurückzukommen ist.

\subsection{Psychologische Orientierung: Zum positiven Sinn "falscher" oder „neurotischer“ Schuldgefühle bei Menschen, die keine wirkliche Schuld haben}

Anhand der eingangs genannten Fallbeispiele hatten wir schon gesehen: Auch in Fällen, in denen es aus ethischer Sicht nicht sinnvoll ist, von „Schuld“ i.S. von Verschulden zu reden, kann die subjektive Schuldkonstruktion des Klienten eine sinnvolle psychologische Funktion haben. Vier solcher Funktionen sind: ${ }^{24}$

1. Schuld als Erklärung für Unbegreifliches (Sinn statt Sinnlosigkeit)

2. Schuldgefühl statt unerträglicher anderer Gefühle (Verschiebung)

3. Schuld (d.h. Macht) statt Ohnmacht

4. Schuld (d.h. Verbundenheit) statt Isolation

zu 1.: Schuld als Erklärung für Unbegreifliches (Sinn statt Sinnlosigkeit)

Wenn ein erschütterndes Widerfahrnis jemandem unbegreiflich und unerklärlich ist und er es nicht in irgendeinen Sinnzusammenhang bringen kann, der ihn das Leben verstehen lässt, steigert das sein Bewältigungsproblem. Wie konnte das passieren? Warum? Man sucht nach Erklärungen. Schuld ist eine mögliche Antwort. Wenn jemand Schuld ist, gibt es einen plausiblen Grund für das Geschehene: Lieber Schuld als Sinnlosigkeit. Schuld ist für viele Menschen leichter auszuhalten als ein sinnloses Leben in einer sinnlosen Welt.

24 Zu weiteren Mechanismen vgl. Paul. C., Schuld - Macht - Sinn. Arbeitsbuch für die Begleitung von Schuldfragen im Trauerprozess. Gütersloh 2010. 


\section{zu 2.: Schuldgefühl statt unerträglicher anderer Gefühle (Verschiebung)}

Ein überwältigendes Widerfahrnis löst starke Gefühle aus: Angst, Wut, Trauer usf. Nicht jedes davon ist jedem Menschen vertraut und erträglich. Wenn ein Schuldgefühl ihnen vertrauter und erträglicher ist, deuten sie ein anderes Gefühl u.U. zu einem Schuldgefühl um: Lieber schuldig als ängstlich, wütend, traurig. Oder: Lieber schuldig als nicht wissen, was ich fühle, unsicher, mich selbst nicht mehr kennend. Der Vorteil ist, dass sie das Schuldgefühl kennen und deshalb besser handhaben können. Ein weiterer Vorteil kann sein, dass ihnen das Schuldgefühl ermöglicht, ein tragfähigeres Selbstkonzept zu erhalten, wie wir gleich sehen werden (s. zu 3.).

zu 3.: Schuld (d.h. Macht) statt Ohnmacht

Wer schrecklichen Widerfahrnis ausgesetzt war und nichts dagegen tun konnte, kann sich als hilflos ausgeliefert und als ohnmächtig erleben. Wird dieses Erleben generalisiert, kann daraus eine lähmende Handlungsunfähigkeit resultieren. ${ }^{25}$ Es handelt sich hierbei jeweils um subjektive Bedeutungsgebungsprozesse (ich interpretiere mich als hilflos, ohnmächtig etc.), die auch anders konstruiert werden können. Eine Alternative dazu kann es sein, sich selbst die Schuld an dem Widerfahrnis zu geben: Lieber schuldig als ohnmächtig, denn wer Schuld hat, hat Macht über das Geschehen. Er hat es ja selbst verursacht. Er ist ihm nicht ausgeliefert, nicht Opfer, sondern Täter. Er kann handeln. Er hat Kompetenz.

\section{zu 4.: Schuld (d.h. Verbundenheit) statt Isolation}

Wer einen Menschen verloren hat, wer von einem Anderen verlassen wurde oder verraten und verkauft, ist zurückgelassen und oft einsam. Schuld aber bindet ihn und den Anderen über die Trennung hinaus aneinander. „Hätte ich mich anders verhalten, hätte er mich nicht verlassen, noch nicht verlassen, nicht so verlassen“ etc., lauten gängige Konstruktionen. Man selbst hält noch am anderen Menschen fest. Oder im obigen Beispiel der an Sterbebett wachenden Frau, die den Moment des Todes ihres Mannes verpasste: Gäbe sie sich nicht die Schuld am einsamen Sterben ihres Mannes, müsste sie sich damit auseinandersetzen, dass er sie beim Sterben vielleicht nicht bei sich haben wollte. Gäbe sie sich nicht die Schuld am einsamen Sterben ihres Mannes, müsste sie spüren, dass sie selbst einsam zurückgeblieben ist.

Was mit diesen kurzen Schlaglichtern deutlich gemacht werden soll, ist:

Auch wenn ein Schuldgefühl oder eine Schuldkonstruktion im Sinne eines ethischen Urteils als „unangemessen“ erscheint - sie können eine andere sinnvolle Funktion für den Klienten haben. Deshalb ist es seelsorglich nicht angezeigt, den Klienten von seinem Schuldgefühl oder seiner Schuldkonstruktion abbringen zu wollen, solange er keine Alternative hat, die diese Funktion anders erfüllt. Man würde ja auch niemandem seine Krücke wegnehmen, solange er nicht wieder ohne sie laufen kann und selber bereit ist, sie abzulegen. Zunächst ist es also seelsorglich angezeigt, nach der Funktion der „Schuld“ zu fragen:

25 Es handelt sich hierbei natürlich um subjektive Bedeutungsgebungsvorgänge bzw. Interpretationen von Erlebnissen, die auch ganz anders gedeutet werden können - alternative, heilsamere Betrachtungsweisen können in seelsorglichen und psychologischen Beratungen eingeführt werden. Zur einer wissenschaftlich Ausdifferenzierung Beschreibung der hier nur kurz benannten Dynamik in Bezug auf Psychotraumatologie und kognitive Stresstheorie vgl. Lammer, Kalter Schweiß auf dem Rücken (vgl. Anm. 21) 
Welches Problem hätte der Klient, wenn er das Schuldproblem nicht hätte? Und welche alternativen Lösungswege gäbe es, dieses Problem zu bearbeiten - außer mittels der Hilfskonstruktion „Schuld“?

Hierzu kommen theologische Konstruktionen oder spirituelle Wege als heilsame Alternativen infrage. Darauf soll im nächsten Abschnitt eingegangen werden.

\subsection{Theologisch-existentiale Orientierung: Das Maß des Mensch- seins - heilsamer Umgang mit Schulderleben}

Schulderleben führt Menschen an ihre existentialen Grenzen.

Die Tatschuld (culpa) führt in die Differenzerfahrung: Man erlebt die eigene Unvollkommenheit. Die eigene Person ist mit sich selbst nicht identisch, sondern voller Ambivalenzen, Brüche und Widersprüche. Diese Erfahrung hat der Apostel Paulus mit den Worten beschrieben hat „... das Gute, das ich tun will, tue ich nicht, sondern das Böse, das ich nicht tun will, das tue ich" (Röm 7,19).

Die Seinsschuld führt in die Kontingenzerfahrung: Man erlebt die Unverfügbarkeit des Lebens. Das ist m.E. das tiefer liegende, weil letztlich spirituelle Problem. Nachstehend möchten wir erläutern, welchen spirituellen Kern die o.a. vier Typen „falscher“ Schuldgefühle haben, bzw. welcher spirituelle Umgang damit heilsam sein kann.

1. Schuld als Erklärung für Unbegreifliches (Sinn statt Sinnlosigkeit) Das spirituelle Problem, das diese Art von Schuldkonstruktion zu lösen versucht, lautet: „Ich kann nicht fassen, was mir passiert ist, ich verstehe es nicht, ich suche einen Grund." Fraglich, ist, ob ein auf Dauer tragender Grund in einer Erklärung (wie z.B.: Schuld) gefunden werden kann, oder letztlich nur in einem tragenden Sinngrund, der seelsorglich zu explorieren wäre.

2. Schuldgefühl statt unerträglicher anderer Gefühle (Verschiebung) Eine Schuldkonstruktion kann eine Antwort auf das spirituelle Problem sein: „Ich kann nicht ertragen, was ich fühle." Fraglich ist, ob eine solche Form der Ab- oder Umlenkung dauerhaft aus diesem Problem erlöst. Eine bessere Alternative könnte sein, dass jemand das unaussprechliche Erleben seelsorglich zur Sprache und zur Darstellung bringt und der betroffene Mensch erfährt: ich bin nicht der oder die Einzige, es gibt unzählige Menschen vor und neben mir, die das Cleiche erlebt haben und es benennen, gestalten und bewältigen konnten. Ich denke hier an die Symbolsprache etwa von Psalmtexten und Ritualen.

3. Schuld (d.h. Macht) statt Ohnmacht

Wenn das spirituelle Problem ist: „Ich fühle mich ohnmächtig“- kann es dann dauerhaft daraus erlösen, mächtig zu agieren? Oder ist es eher entlastend, die eigenen Grenzen und Möglichkeiten, das Maß des eigenen Menschseins realistisch einzuschätzen und damit Frieden zu machen?

Dieser Punkt soll noch ein wenig vertieft werden; nach unserem Dafürhalten sind wir hier beim Kern der Sache: ${ }^{26}$

$26 \mathrm{Vgl.} \mathrm{zu} \mathrm{den} \mathrm{folgenden} \mathrm{beiden} \mathrm{Abschnitten} \mathrm{ausführlicher:} \mathrm{Lammer,} \mathrm{K.,} \mathrm{„Lehre} \mathrm{uns} \mathrm{bedenken,} \mathrm{dass} \mathrm{wir} \mathrm{sterben} \mathrm{müssen,} \mathrm{damit} \mathrm{wir}$ ein weises Herz gewinnen", in: Nittel, Dieter/Seltrecht, Astrid, Krankheit: Lernen im Ausnahmezustand? Brustkrebs und Herzinfarkt in interdisziplinärer Perspektive, Berlin/Heidelberg 2013, S. 545-552. 
Schuld ist, genau wie das Leid, eine Grenzerfahrung (das verbindet übrigens Täter und Opfer). Wer leidet und wer schuldig wird, stößt an seine Grenzen. Die Erfahrung der Grenze und der eigenen Begrenztheit ist real, die subjektive Interpretation als „Ohnmacht“ ist aber irreal, d.h. eine Fehlinterpretation. Aus psychologischer Sicht handelt es sich um eine Ohnmachtsphantasie, die erst am enttäuschten Allmachtswunsch entsteht. Man hat sich vorher als rein und unschuldig, als heil und ganz phantasiert, und nun, wo die Illusion der Ganzheit verloren geht, schlägt sie um in die Phantasie, man habe sich ganz verloren. Als sei man alles Sinnvollen, aller Fähigkeiten und Möglichkeiten, aller Rechte beraubt - ohnmächtig. Beide, Allmachts- und Ohnmachtsphantasie, sind aber unrealistisch. Die Chance der Grenzerfahrung ist es, beide aufzugeben und statt dessen zu einer realistischen Selbsteinschätzung zu kommen:

Psychologisch gesprochen, akzeptiert die reife Persönlichkeit ihre Begrenzungen, ergreift aber entschlossen ihre Möglichkeiten. Theologisch gesprochen, nimmt, wer glaubt, das von Gott geschenkte Leben als Gabe und als Aufgabe an, entfaltet nach Kräften die eigenen Möglichkeiten, verantwortet und überantwortet/verwindet sein Scheitern. „Praxissituation endlicher Freiheit“ nennt der evangelische Dogmatiker Eilert Herms das.

Dass das Stoßen an Grenzen nicht vorkommen dürfe oder dass es der Ereignisbruch sei, der den betroffenen Menschen von den anderen trenne und unterscheide, ist falsch. Damit sind wir beim letzten Punkt:

\section{Schuld (d.h. Verbundenheit) statt Isolation}

Wenn hinter einer Schuldkonstruktion als spirituelle Aussage steht: „Das hätte nicht passieren dürfen. Warum passiert das gerade mir?“, dann ist das Ausdruck der falschen Vorstellung, das Stoßen an existenzielle Grenzen sei ein Ausnahmefall. Das trifft ja nicht zu, im Gegenteil: Es ist der Regelfall, weil die Kontingenz zu den Systembedingungen der Existenz gehört. Nichts ist im Leben sicher, außer, dass wir an unsere Grenzen kommen. Diese Einsicht schließt auch die Einsicht in die kreatürliche Angewiesenheit auf andere ein: Ich werde geboren, ich werde geliebt, ich werde gepflegt, mir wird vergeben. Das Passiv zeigt hier an: Alles, was im Leben wirklich wichtig ist, kann ich mir nicht selber geben, ich kann es mir nicht nehmen, ich kann es mir nur schenken lassen. Wir sind angewiesen auf ein Du: auf seine Liebe, auf seine Zuwendung, auf seine Vergebung. Alle. Immer. Die Chance an Grenzerfahrungen wie Leid und Schuld ist, dass wir uns das bewusst machen und bereit werden, Zuwendung und Vergebung zu gewähren und anzunehmen.

Wir sind angewiesen auf die anderen. Deshalb ist das Wichtigste, was wir Leidenden und Schuldigen zu geben haben, das Sakrament der Gemeinschaft. Es drückt aus: Du wurdest verletzt und du hast verletzt. Du wirst verletzt werden und du wirst verletzen. Aber du wirst nicht verlassen.

Es gibt in diesem Leben keine Hoffnung auf Unversehrtheit. Es gibt in diesem Leben auch keine Hoffnung auf Unschuld. Aber es gibt die Hoffnung auf Gemeinschaft und Verbundenheit. Dies zu entdecken und dessen getrost zu werden, könnte ein heilsamer spiritueller Weg sein, Schulerleben zu bewältigen . 


\subsection{Interprofessionelle Orientierung: Zu Rolle und Funktion der Seelsorge}

Die o.a. Hoffnung auf Cemeinschaft und Verbundenheit tragen Seelsorgende in die Notfallbegleitung ein. Von ihrer professionellen Rolle her verweisen sie auch auf das Thema der Verbundenheit, der Gemeinschaft mit Gott - und zwar schon ehe und ohne dass sie davon reden oder überhaupt irgendetwas tun oder sagen. Denn sie sind Symbolfiguren für das religiöse Thema. Ihre pure Anwesenheit verweist auf die christliche Hoffnung, die heißt: Du bist nicht heil, sondern gebrochen. Du bist nicht heilig, sondern schuldig. Aber du bist gesegnet, und nicht verflucht.

Was ändert sich dadurch? Die Haltung. Anders betrachtet: der haltende Rahmen. Im Sinne der systemischen Beratung kann man das auch als Reframing verstehen. Man legt sich „Gegebenheiten, die nicht zu ändern sind, so zurecht, dass sich mit ihnen leben lässt“". ${ }^{27}$ Wir rücken das Unabänderliche in eine Perspektive, die es uns ermöglicht, uns dazu zu verhalten. Wir fragen, wie sich mit Leiden und Schuld leben lässt, und was unser Leben dennoch zusammenhält. Wir finden eine Einstellung, mit der sich weiter leben lässt. Christen nennen das „Glauben“.

Das zuvor in seelsorglicher Perspektive Gesagte sei hier zusammengefasst:

Schuld und Leid sind unvermeidlich. Sie sind Begegnungen mit der Kontingenz. Aufgabe der Seelsorge ist nicht, sie zu ermäßigen, sondern sie zu thematisieren. Das wichtigste Heilsmittel, das Seelsorgende dabei weiterzutragen haben, ist das Sakrament der Gemeinschaft. Gemeinschaft lässt die eigenen Grenzen ertragen und transzendiert sie. Die Grenze kann annehmbar werden.

Wer anderen glaubwürdig zur Akzeptanz der eigenen Grenzen verhelfen will, muss sich selbst auf einen spirituellen Weg des Umgangs mit den eigenen Grenzen machen. Das schließt das Bewusstsein um die Grenzen der eigenen Möglichkeiten als professionell handelnder Mensch ein: Auch Seelsorgende können die Schuld und das Leid ihrer Klienten nicht wegnehmen - das ist Gottes Metier, nicht menschliches. Auch sie müssen die eigenen Allmachts- und Ohnmachtsphantasien, das eigene Sein-Wollen-wie-Gott loslassen. Sie dürfen die eigene Angst um die Annehmbarkeit ihres Könnens und Tuns in der Seelsorge nicht kompensieren, indem sie immer mehr zu leisten und zu machen versuchen. Im Gegenteil: Im Unterschied zu allen anderen Professionen im Notfalleinsatz ist es gerade nicht die Aufgabe als Seelsorgenden, zu tun und zu machen, Probleme weg zu machen, sondern die spezielle Aufgabe Seelsorgender ist es, zu sein und sein zu lassen. Sie sind nicht die Do-ers, sondern die Beers. Es ist anstrengende seelische Arbeit, die Dinge und die Menschen so sein zu lassen, wie sie sind (nicht, wie sie sein sollten), und die Grenzerfahrung eines schlimmen Widerfahrnisses zu akzeptieren. Eine realistische Einschätzung und eine getroste Haltung $\mathrm{zu}$ unseren eigenen Grenzen und Möglichkeiten in unserem professionellen Handeln zu finden, ist auch ein Werk der Stellvertretung. 


\section{Literatur}

Auchter T (1996) Von der Unschuld zur Verantwortung. In: Schlagbeck T (Hrsg.) Theologie und Psychologie im Dialog über Schuld. Paderborn, S. 41-138

Bobert S (2004) Fremder Schuld geopfert sein. Wege zum Menschen 56(5): 421-435

Dalfert I (2007) Leiden und Böses. Vom schwierigen Umgang mit Widersinnigem. 2. Aufl. Leipzig

Funke D (2000) Das Schulddilemma. Wege zu einem versöhnten Leben. Göttingen

Härle W (2007) Dogmatik, Kap. Die gefallene Welt. Hamartiologie, Berlin, S. 456-492

Klessmann M (2004) Pastoralpsychologie. Ein Lehrbuch. Neukirchen-Vluyn

Körtner U (2006) Sündenvergebung und Schuldübernahme in der Seelsorge. WzM 58: 259-269

Lammer K (2013) Lehre uns bedenken, dass wir sterben müssen, damit wir ein weises Herz gewinnen. In: Nittel D, Seltrecht A (Hrsg.) Krankheit: Lernen im Ausnahmezustand? Brustkrebs und Herzinfarkt in interdisziplinärer Perspektive. Berlin/Heidelberg, S. 545-552.

Paul C (2010) Schuld - Macht - Sinn. Arbeitsbuch für die Begleitung von Schuldfragen im Trauerprozess. Gütersloh

Reichmann A (2010) „Das gute Gewissen ist eine Erfindung des Teufels.“ Ein anderer Umgang mit Schuld und Vergebung als Auftrag der Kirche. Vortrag am 24.11.2010 im Altonaer PastorInnenkonvent, http://www. pastoralpsychologie-norden.de/fileadmin/_pastoralpsychologie/publikationen/Schuld_und_Vergebung.pdf.

Schulze G (2006) Die Sünde. Das schöne Leben und seine Feinde. München/Wien

Schweitzer A (1923) Kultur und Ethik (Kulturphilisophie, Bd. II). München, Bern

Sievenich M (2005) Schuld und Vergebung. Grundthema und Anfrage. WzM 57: 298-308

Sitzler-Osing D, van den Beld A, Genest H (1999) Art. Schuld. TRE 30: 572-590

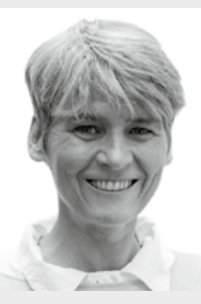

\section{Prof. Dr. Kerstin Lammer}

Theologin und Pastoralpsychologin, Supervisorin/Lehrsupervisorin (DGSv/ DGfP), Systemische Familientherapeutin (SG). Berufliche Tätigkeiten als Krankenhausseelsorgerin in USA, Beratungspastorin in einer Einrichtung für Menschen mit geistigen Behinderungen, Gemeindepfarrerin, Weiterbildnerin, Supervisorin und Therapeutin.

Seit 2007 Professorin für Seelsorge und Pastoralpsychologie, Leiterin des MAStudiengangs Supervision an der Evangelischen Hochschule Freiburg. Vorsitzende der Ständigen Konferenz für Seelsorge in der EKD. 